\section{Prevalência de Lesão Adrenal Incidental em Pacientes Submetidos a Tomografia Computadorizada de Tórax e Abdome em Um Hospital Geral Brasileivo}

\section{RESUMO}

Introdução: Determinar prospectivamente a prevalência de lesão adrenal incidental a tomografia computadorizada (TC) em um hospital geral universitário brasileiro. Pacientes e Método: Estudo transversal, prospectivo, avaliando pacientes consecutivos sem doença adrenal conhecida, submetidos a TC de tórax e abdome no Hospital de Clínicas de Porto Alegre, em um período de 10 meses. Incidentalomas adrenais foram definidos pela presença de alteração no contorno, tamanho ou densidade da glândula. Valores de kappa foram calculados, avaliando a concordância intra e inter-observador. Resultados: Identificaram-se 101 lesões adrenais incidentais em 83 de 3.382 pacientes, correspondendo a uma prevalência de $2,5 \%$ (IC 95\%: $1,0 \%-3,2 \%$ ). A prevalência de lesões adrenais aumentou com a idade e foi significativamente mais alta em pacientes do sexo masculino (3,3\% vs. $1,5 \% ; p=0,01)$. Na avaliação das concordâncias intra e interobservador, obteve-se um kappa de 0,76 (boa concordância) e 0,83 (excelente concordância) respectivamente. Conclusões: A prevalência de incidentaloma adrenal detectada por TC foi semelhante à de diversas séries clínicas retrospectivas publicadas. Os dois fatores que influenciaram de forma independente a distribuição dos incidentalomas foram aumento na idade e sexo masculino. (Arq Bras Endocrinol Metab 2005;49/5:769-775)

Descritores: Incidentaloma adrenal; Tomografia computadorizada; Prevalência

\section{ABSTRACT}

Prevalence of Adrenal Incidentaloma at Computed Tomography (Chest and Abdominal) in a General Hospital in Brazil.

Objective: To prospectively determine the prevalence of incidental adrenal lesions at computed tomography (CT) at an University Hospital in Brazil. Patients and Method: A cross-sectional prospective study was performed to evaluate 3,382 consecutive patients with no known adrenal disease or malignancies who underwent chest and abdominal CT scans over a ten-month period. The cases of adrenal incidentaloma were defined by the presence of any change in gland contour, size or density. Kappa values were calculated, evaluating the intra and inter-observer agreement. Results: One hundred and one incidental adrenal masses were identified, corresponding to 83 cases and a prevalence of $2.5 \%(\mathrm{Cl}$ 95\%: $1.0 \%-3.2 \%$ ). Male patients presented a higher prevalence of incidentaloma than female $(3.3 \%$ vs. $1.5 \% ; p=0.01)$. There was a significant association between older age and higher frequency of incidentaloma. Kappa of 0.76 and 0.83 (good and excellent agreement, respectively), were obtained in evaluating the intra and inter-observer agreements. Conclusions: The prevalence of adrenal incidentaloma detected by CT was similar to that of various published retrospective clinical series. The two factors that independently influenced the distribution of incidentalomas were older age and being male. (Arq Bras Endocrinol Metab 2005;49/5:769-775)

\section{artigo original}

\author{
Eleci V. Ferreiva \\ Mauro A. Czepielewski \\ Carlos Sasso Faccin \\ Melissa Coral Accordi \\ Álvaro Porto Alegre Furtado
}

Serviços de Radiologia (EVF, CSF, MCA, APAF) e

Endocrinologia (MAC) do Hospital de Clínicas de Porto Alegre, Programa de Pós-Graduação em Ciências Médicas: Endocrinologia, da

Faculdade de Medicina, UFRGS, Porto Alegre, RS.

Recebido em 14/05/05

Revisado em 20/06/05 Aceito em 20/06/05 
Keywords: Adrenal incidentaloma; Computed tomography; Prevalence

LESÕES ADRENAIS CLINICAMENTE silenciosas, descobertas durante a investigação ou tratamento de condições não relacionadas à suspeita de doença adrenal, são definidas como incidentalomas e representam um desafio na prática médica, pois podem corresponder a um achado destituído de significado clínico ou determinar altas taxas de morbidade e mortalidade, quando decorrentes de lesões funcionantes ou malignas (1-3).

A prevalência de incidentaloma adrenal (IA) ainda não está bem definida, possivelmente variando conforme os critérios de inclusão no estudo, a faixa etária da população, a indicação clínica para o exame de imagem e o método utilizado para o diagnóstico nas diferentes séries. Estudos de necropsia identificaram a presença de tumores adrenais entre 1,0\% e $9,0 \%$ dos pacientes sem evidência de disfunção hormonal antes do óbito $(3,4)$. Em estudos de tomografia computadorizada (TC), a prevalência oscilou entre $0,4 \%$ e $4,4 \%$ (5). Entretanto, os dados epidemiológicos disponíveis na literatura são provenientes, em sua maioria, de séries retrospectivas, muitas delas com reduzido número de pacientes, e que utilizaram equipamentos com menor resolução em comparação aos disponíveis atualmente. O desenvolvimento de métodos radiológicos com melhor definição de imagem e sua aplicação em larga escala têm contribuído para o aumento do número de diagnósticos não-invasivos de IA (3).

O objetivo deste estudo foi avaliar prospectivamente a prevalência de incidentaloma adrenal nos pacientes sem história de doença adrenal submetidos a TC de abdome e tórax, em um hospital universitário do sul do Brasil.

\section{MATERIAL E MÉTODOS}

\section{Delineamento e local de realização do estudo}

Estudo transversal, prospectivo, realizado na Unidade de Tomografia Computadorizada do Serviço de Radiologia do Hospital de Clínicas de Porto Alegre (HCPA), hospital geral universitário com 810 leitos, com uma média mensal de 2.227 internações e de 39.400 consultas ambulatoriais (6).

\section{Aspectos éticos}

Previamente à sua realização, o estudo foi submetido a avaliação pelo Comitê de Ética do Grupo de
Pesquisa e Pós-Graduação do HCPA, tendo sido assinado o Termo de Compromisso para Utilização de Dados.

\section{Pacientes}

Todos os pacientes encaminhados para realização de TC de tórax ou abdome na Unidade de TC do HCPA, entre janeiro e outubro de 2001, foram avaliados para inclusão no estudo. Foram excluídos os casos cuja técnica do exame não permitia a avaliação adequada das adrenais (TC com técnica de alta resolução, TC com ênfase em estruturas pélvicas e angiotomografia) e aqueles com suspeita clínica ou diagnóstico de lesão adrenal estabelecido previamente ao exame.

Os dados demográficos e clínicos dos pacientes, como idade, sexo, procedência, suspeita ou diagnóstico clínico que motivou a realização da TC, foram obtidos a partir dos formulários preenchidos rotineiramente pelos médicos assistentes, no momento da solicitação do exame.

\section{Tomografia computadorizada}

Os exames foram realizados aleatoriamente em dois tomógrafos espirais, o Somatom Plus 4, Siemens Alemanha (1997), e o Elscint Twin, Philips - Estados Unidos da América (1997). Os parâmetros técnicos utilizados habitualmente na Unidade de TC do HCPA estão dispostos no quadro 1 .

As TCs de abdome incluíram fases com e sem injeção intravenosa do meio de contraste. Nas TCs de tórax, o uso do meio de contraste foi individualizado de acordo com a suspeita clínica ou alterações identificadas durante a aquisição das imagens. Para a realização de TC de abdome, meio de contraste iodado diluído foi administrado por via oral. O fluxo de injeção do meio de contraste foi de 1,0 a $2,0 \mathrm{ml} / \mathrm{s}$, por acesso venoso periférico, através de bomba injetora modelo Med Rad En Vision CT Injector, no aparelho Siemens, e modelo Med Rad Op 100, no aparelho Philips, com um tempo de retardo médio de 20

Quadro 1. Parâmetros técnicos espirais utilizados em tomografias computadorizadas (TC) de tórax e abdome.

\begin{tabular}{lcc}
\hline PARAMETRO & TC DE TÓRAX & TC DE ABDOME \\
\hline KV & 120 & $140^{\star} / 120^{\star *}$ \\
$\mathrm{~mA}$ & $160^{\star} / 200^{\star *}$ & $240^{\star} / 200^{\star *}$ \\
Filtro & $\mathrm{AB} 50^{\star} / \mathrm{C}^{\star *}$ & $\mathrm{AB} 30^{\star} / \mathrm{B}^{\star *}$ \\
Espessura do corte & $8 \mathrm{~mm}$ & $8 \mathrm{~mm}$ \\
Intervalo do corte & $8 \mathrm{~mm}$ & $8 \mathrm{~mm}$ \\
Pitch & $1,0^{\star} / 0,7^{\star *}$ & $1,0^{*} / 0,7^{\star *}$ \\
\hline * Parâmetros utilizados com tomógrafo da Siemens $\mathrm{e}^{\star *}$ \\
da Philips.
\end{tabular}
da Philips. 
segundos entre o início da injeção e o início dos cortes tomográficos, com uma dose de 1,5 a $2,0 \mathrm{ml} / \mathrm{kg}$.

As adrenais foram examinadas sempre pelo mesmo radiologista, com ênfase na avaliação do tamanho da glândula em três eixos (ântero-posterior, transversal e craniocaudal, quando possível), contornos, densidade, impregnação ao meio de contraste intravenoso e presença de calcificações.

Dez meses após a avaliação inicial, os registros médicos de todos os pacientes com diagnóstico de incidentaloma foram revisados para verificação da conduta assumida pela equipe assistente quanto à investigação do caso e diagnóstico final da lesão adrenal.

\section{Análise estatística}

Os casos de IA foram definidos pela presença de alteração no contorno, tamanho ou densidade da glândula, sendo a prevalência descrita em percentual, com cálculo do intervalo de confiança de 95\% (IC 95\%). As variáveis categóricas foram comparadas utilizando-se o teste do qui-quadrado (teste de Pearson), sendo empregado um valor de $\mathrm{p}<0,05$ como nível de significância estatística. Posteriormente, foi aplicado um modelo de regressão logística para análise da estimativa do risco de IA em relação ao sexo, idade, procedência e diagnóstico de neoplasia.

Dados referentes às características radiológicas das lesões adrenais foram apresentados de forma descritiva.

Valores de kappa foram calculados em 100 pacientes, avaliando a concordância entre a interpretação

Tabela 1. Características demográficas e clínicas dos 3.382 pacientes estudados.

\begin{tabular}{lc}
\hline VARIAVEL & VALOR \\
\hline $\begin{array}{l}\text { Idade (anos)* } \\
\text { Sexo }\end{array}$ & $50,2 \pm 20,4$ \\
Masculino & $1.803(53,3 \%)$ \\
Feminino & $1.579(46,7 \%)$ \\
Procedência & \\
Ambulatório & $2.106(62,3 \%)$ \\
Internação & $1.276(37,7 \%)$ \\
Tipo de Exame & \\
Abdome & $1.907(56,4 \%)$ \\
Tórax & $930(27,5 \%)$ \\
Tórax e abdome & $545(16,1 \%)$ \\
Motivo do Exame & \\
Investigação/Estadiamento & \\
de Neoplasia & $1.785(52,7 \%)$ \\
Doença Gastrintestinal benigna & $455(13,5 \%)$ \\
Doença Pulmonar benigna & $446(13,2 \%)$ \\
Doença Infecciosa & $170(5,0 \%)$ \\
Doença Cardiovascular & $149(4,4 \%)$ \\
Doença Geniturinária benigna & $100(3,0 \%)$ \\
Outras & $277(8,2 \%)$ \\
Diagnóstico Confirmado de Neoplasia & $1.756(51,9 \%)$ \\
\hline *Média \pm desvio-padrão. &
\end{tabular}

do radiologista responsável pelo estudo e um radiologista independente convidado, assim como a concordância intra-observador com um intervalo de dez meses.

\section{RESULTADOS}

Entre janeiro e outubro de 2001, foram realizadas 3.802 TC de tórax e abdome. Foram excluídos 420 casos, 96 por haver suspeita ou diagnóstico estabelecido de lesão adrenal e 324 por a técnica de exame não permitir adequada avaliação das adrenais (100 TCs de tórax com técnica de alta resolução e 224 TCs de tórax e abdome sem inclusão das adrenais).

Assim, foram incluídos no estudo 3.382 pacientes, sendo 1.803 (53\%) homens e 1.579 (47\%) mulheres, com média de idade de 50,2 $\pm 20,4$ anos. Em relação à técnica de exame, $1.907(56,4 \%)$ casos foram provenientes de TC de abdome, $930(27,5 \%)$ de TC de tórax e $545(16,1 \%)$ de TC de tórax e abdome. As características clínicas e demográficas dos pacientes estão demonstradas na tabela 1 .

Identificaram-se 83 casos de IA, correspondendo a uma prevalência de 2,5\% (IC 95\%: 1,0\%-3,2\%) e a um total de 101 lesões adrenais incidentais, sendo $45(54,2 \%)$ à esquerda, $20(24,1 \%)$ à direita e $18(21,7 \%)$ bilaterais. A prevalência de IA foi 3,6\% nas TCs de tórax e abdome, 2,2\% nas TCs de abdome e 2,1\% nas TCs de tórax.

Tabela 2. Prevalência de incidentaloma adrenal em relação a sexo, idade, diagnóstico de neoplasia e internação hospitalar.

\begin{tabular}{lccc}
\hline VARIÁVEL & $\begin{array}{c}\text { NÚMERO DE } \\
\text { PACIENTES }\end{array}$ & $\begin{array}{c}\text { NÚMERO DE } \\
\text { CASOS (\%) }\end{array}$ & P \\
\hline $\begin{array}{c}\text { Sexo } \\
\text { Masculino }\end{array}$ & 1.803 & $59(3,3)$ & 0,01 \\
$\quad \begin{array}{l}\text { Feminino } \\
\text { Faixa Etária }\end{array}$ & 1.579 & $24(1,5)$ & \\
$\quad$ 60 anos & 1.275 & $48(3,8)$ & $<0,01$ \\
30-59 anos & 1.528 & $31(2,0)$ & \\
0-29 anos & 579 & $4(0,7)$ & \\
Diagnóstico de Neoplasia & & \\
$\quad$ Sim & 1.756 & $48(2,7)$ & 0,28 \\
$\quad$ Não & 1.626 & $35(2,2)$ & \\
Procedência & & & \\
Internação & 1.276 & $39(3,1)$ & 0,08 \\
$\quad$ Ambuláório & 2.106 & $44(2,1)$ & \\
\hline
\end{tabular}

Tabela 3. Análise de regressão logística para estimativa de risco de incidentaloma adrenal. *

\begin{tabular}{|c|c|c|}
\hline VARIAVEL & ODDS RATIO (IC 95\%) & $\overline{\mathbf{P}}$ \\
\hline Sexo (masculino) & $2,06(1,27-3,33)$ & $<0,01$ \\
\hline Idade & $1,03(1,02-1,04)$ & $<0,01$ \\
\hline Neoplasia & $1,34(0,86-2,10)$ & 0,20 \\
\hline Internação hospitalar & $1,48(0,95-2,30)$ & 0,09 \\
\hline
\end{tabular}
categóricas; idade é variável contínua. 
Os pacientes do sexo masculino apresentaram maior prevalência de IA do que os do sexo feminino (3,3\% vs. $1,5 \% ; p=0,01)$, o que corresponde a uma odds ratio (OR) de 2,06\% (IC 95\%: 1,27-3,33). Ocorreu associação significativa entre aumento da idade e maior freqüência de IA, com uma OR de 1,03 (IC 95\%: 1,02-1,04) para cada ano de vida $(\mathrm{p}<0,01)$. Não houve diferença na prevalência de lesão adrenal entre os pacientes com ou sem diagnóstico de neoplasia $(2,7 \%$ vs. $2,2 \% ; \mathrm{p}=0,28)$, bem como entre a procedência da internação ou do ambulatório (3,1\% vs. $2,1 \% ; p=0,08)$. Após a análise de regressão logística, considerando-se todas as variáveis, as relações se mantiveram constantes (tabelas 2 e 3 ).

A tabela 4 expõe as características radiológicas observadas nas lesões adrenais incidentais dos 83 pacientes. Nos casos de lesão bilateral, elas foram definidas pela presença de anormalidade em qualquer uma das glândulas. Em relação ao tamanho das lesões, 21 $(25,3 \%)$ pacientes apresentavam lesão com maior eixo inferior a $3,0 \mathrm{~cm}, 53(63,9 \%)$ com lesão com maior eixo entre 3,0 e $5,0 \mathrm{~cm}$ e $9(10,8 \%)$ com lesão com maior eixo superior a $5,0 \mathrm{~cm}$. Quanto à densidade da lesão na fase não contrastada do exame, em 15 (18,1\%) pacientes ela era inferior a 10 unidades Hounsfield

Tabela 4. Características radiológicas das lesões adrenais dos 83 casos de incidentaloma. *

\begin{tabular}{|c|c|}
\hline CARACTERISTICA & NUMERO DE PACIENTES (\%) \\
\hline \multicolumn{2}{|l|}{ Lateralidade } \\
\hline Adrenal direita & $20(24,1)$ \\
\hline Adrenal esquerda & $45(54,2)$ \\
\hline Bilateral* $^{*}$ & $18(21,7)$ \\
\hline \multicolumn{2}{|l|}{ Contorno } \\
\hline Regular & $71(85,5)$ \\
\hline Irregular & $12(14,5)$ \\
\hline \multicolumn{2}{|l|}{ Tamanho } \\
\hline$<3,0 \mathrm{~cm}$ & $21(25,3)$ \\
\hline $3,0-5,0 \mathrm{~cm}$ & $53(63,9)$ \\
\hline$>5,0 \mathrm{~cm}$ & $9(10,8)$ \\
\hline \multicolumn{2}{|l|}{ Densidade** } \\
\hline$<10 \mathrm{UH}$ & $15(18,1)$ \\
\hline $10-20 \mathrm{UH}$ & $13(15,7)$ \\
\hline$>20 \mathrm{UH}$ & $55(66,2)$ \\
\hline \multicolumn{2}{|c|}{ Impregnação pelo meio de contraste } \\
\hline Heterogênea & $14(16,9)$ \\
\hline Homogênea & $35(42,1)$ \\
\hline Ausente & $34(41,0)$ \\
\hline \multicolumn{2}{|l|}{ Calcificações } \\
\hline Presentes & $7(8,4)$ \\
\hline Ausentes & $76(91,6)$ \\
\hline \multicolumn{2}{|c|}{$\begin{array}{l}\text { * Nos } 18 \text { casos de lesão bilateral, foi considerada a lesão } \\
\text { com caractere positivo: contorno irregular, maior } \\
\text { tamanho e densidade, impregnação heterogênea e } \\
\text { calcificações presentes. } \\
\text { ** Densidade da adrenal medida na fase pré-contraste } \\
\text { em unidades Hounsfield. }\end{array}$} \\
\hline
\end{tabular}

(UH), em $13(15,7 \%)$ se situava entre 10 e $20 \mathrm{UH}$ e em $55(66,2 \%)$ era superior a $20 \mathrm{UH}$.

Biópsia adrenal percutânea aspirativa por agulha fina foi utilizada na investigação de $19(22,9 \%)$ pacientes, $6(7,2 \%)$ foram submetidos a laparotomia e 5 $(6,0 \%)$ foram encaminhados a necropsia. Os demais pacientes realizaram apenas avaliação clínico-laboratorial, sendo que 15 destes, ao final do estudo, não possuíam diagnóstico suspeito ou confirmado da lesão adrenal incidental.

$\mathrm{Na}$ avaliação das concordâncias intra e interobservador, obteve-se um kappa de 0,76 (boa concordância) e 0,83 (excelente concordância) respectivamente.

Dentre os 83 pacientes com IA, 39 (47\%) apresentavam hipertensão arterial sistêmica (HAS), 19 $(23 \%)$ diabete melito (DM) e 10 (12\%) obesidade.

Quanto à avaliação laboratorial, dosagem sérica de potássio foi realizada em todos os pacientes com IA, em 13 (15,7\%) foi medido o cortisol após $1 \mathrm{mg}$ de dexametasona, em $30(36,1 \%)$, o sulfato de deidroepiandrosterona (S-DHEA) e em 9 (10,8\%), as metanefrinas urinárias.

Quatro dos 83 pacientes incluídos na presente amostra, apresentavam lesões pulmonares compatíveis com tuberculose à punção aspirativa. A calcificação completa de ambas as adrenais nos dois pacientes impediu a punção aspirativa das mesmas. $\mathrm{O}$ resultado da punção aspirativa das lesões pulmonares e o aspecto tomográfico das lesões adrenais levou à hipótese de tuberculose adrenal.

Na tabela 5 estão apresentados os diagnósticos etiológicos observados ao final do estudo, conside-

Tabela 5. Diagnóstico dos 83 casos de incidentaloma adrenal ao final do estudo.

\begin{tabular}{lc}
\hline DIAGNÓSTICO & $\begin{array}{c}\text { NÚMERO DE } \\
\text { PACIENTES (\%) }\end{array}$ \\
\hline Diagnóstico com Confirmação Histológica & $30(36,1)$ \\
Lesão Metastática & $19(22,9)$ \\
Neoplasia de pulmão & $11(13,3)$ \\
Linfoma & $4(4,8)$ \\
Neoplasia de mama & $2(2,4)$ \\
Melanoma & $2(2,4)$ \\
Adenoma & $6(7,2)$ \\
Neoplasia de rim com invasão de adrenal & $2(2,4)$ \\
Lesão em órgão adjacente & $2(2,4)$ \\
Feocromocitoma & $1(1,2)$ \\
Diagnóstico Clínico-Laboratorial & $38(45,8)$ \\
Lesão Metastática & $23(27,8)$ \\
Adenoma & $8(9,6)$ \\
Tuberculose & $4(4,8)$ \\
Feocromocitoma & $2(2,4)$ \\
Cisto & $1(1,2)$ \\
Sem diagnóstico & $15(18,1)$ \\
Total & $83(100,0)$ \\
\hline
\end{tabular}


rados a partir das conclusões obtidas pelas equipes assintenciais. Dos 83 casos de IA, em $30(36,1 \%)$ se obteve diagnóstico com confirmação histológica, predominando a lesão metastática $(19 / 83: 22,9 \%)$ e os adenomas (6/83: 7,2\%). Em 38/83 (45,8\%) dos casos estabeleceu-se diagnóstico clínico-laboratorial, predominando o achado de lesões metastáticas $(23 / 83$ : $27,8 \%)$, adenomas $(8 / 83: 9,6 \%)$ e tuberculose $(4 / 83$ : $4,8 \%)$. Em 15 casos (18\%) não se obteve diagnóstico final. Entre os 83 casos, observamos 3 pacientes com feocromocitoma $(3 / 83: 3,6 \%)$.

\section{DISCUSSĀO}

Nos últimos anos, tem crescido o interesse pela avaliação das lesões adrenais incidentais. Com a maior e melhor utilização dos métodos de imagem, postula-se que tais lesões sejam encontradas com maior freqüência (1,7-12). Neste estudo, a prevalência de IA por TC foi de $2,5 \%$, resultado semelhante ao de diversas séries clínicas retrospectivas publicadas previamente (1,2,4,5,13-16). Em estudos de necropsias, a prevalência de IA é mais alta, provavelmente devido à não identificação das pequenas lesões pelos métodos disponíveis atualmente $(4,17)$.

Neste estudo, foram incluídas as TCs de tórax que permitiram a avaliação das adrenais, observando-se uma prevalência de IA similar à registrada nos casos de TC de abdome $(2,1 \%$ vs. $2,2 \%)$. Entretanto, a limitação das evidências disponíveis no estudo impede uma recomendação geral quanto à avaliação de adrenais nas tomografias de tórax.

No presente estudo, os dois fatores que influenciaram de forma independente a distribuição dos IA foram aumento na idade e sexo masculino. A idade, como documentado em várias séries, correlaciona-se diretamente com a freqüência de lesão adrenal incidental. É bem conhecido que o IA é pouco freqüente abaixo dos trinta anos de idade e aumenta progressivamente em adultos e idosos (3), o que poderia indicar maior ocorrência de neoplasia nessas faixas etárias, crescimento compensatório em função do dano isquêmico local da aterosclerose ou meramente refletir um número superior de procedimentos diagnósticos realizados nesse grupo de pacientes $(1,18)$.

Em algumas séries radiológicas, a maioria dos pacientes com lesão adrenal incidental é do sexo feminino, o que provavelmente corresponda a um viés de seleção, refletindo o maior número de mulheres que se submetem a exames de imagem, especialmente por doenças biliares $(1,7,10,13)$. Na presente amostra, 53,3\% dos pacientes eram homens. Mesmo após o controle para os possíveis vieses de confusão, encontrou-se um risco estimado em 2,1 vezes maior para os homens apresentarem IA em relação às mulheres $(\mathrm{p}=0,002)$. Esse achado se opõe aos das séries de casos anteriormente referidas. Entretanto, cabe salientar que estudos de necropsia ou de exames gerais de saúde não demonstram qualquer diferença entre os sexos na distribuição dos incidentalomas adrenais $(1,7,10,13)$.

Contrariando os dados epidemiológicos disponíveis (3), não foi aqui observada maior prevalência de IA nos pacientes com diagnóstico de neoplasia em comparação com aqueles que não apresentavam esta condição $(2,7 \%$ vs. $2,2 \%, \mathrm{p}=0,29)$.

As dimensões e a densidade pré-contraste da lesão adrenal incidental são, até o momento, as características mais valorizadas para a decisão terapêutica.

Lesões com mais de 5,0 ou $6,0 \mathrm{~cm}$ devem ser excisadas, ao passo que as com menos de $3,0 \mathrm{~cm}$ podem ser consideradas benignas $(1,3)$. Dentre os 83 pacientes com IA neste estudo, 25,3\% apresentavam lesões cujo maior eixo era inferior a $3,0 \mathrm{~cm}, 63,9 \%$ entre 3,0 e $5,0 \mathrm{~cm}$ e $10,8 \%$ superior a $5,0 \mathrm{~cm}$.

$\mathrm{O}$ córtex adrenal e alguns tumores adrenocorticais benignos contêm gordura intracitoplasmática, conferindo densidades baixas a TC. Densidades baixas, inferiores a $10 \mathrm{UH}$, têm alta especificidade para lesão benigna, porém baixa sensibilidade, enquanto valores entre 15 e $20 \mathrm{UH}$ mostram alta sensibilidade e baixa especificidade $(3,19)$. No presente estudo, 18,1\% dos pacientes apresentaram lesão com densidade máxima inferior a $10 \mathrm{UH}, 15,7 \%$ entre 10 e $20 \mathrm{UH}$ e $66,2 \%$ superior a $20 \mathrm{UH}$.

Uma avaliação laboratorial mínima é também importante na decisão terapêutica, uma vez que a disfunção hormonal subclínica tem implicações reconhecidas na morbimortalidade. Na presente amostra, foi observada uma grande heterogeneidade nas diferentes possibilidades de investigação laboratorial adrenal disponíveis, pois as condutas assumidas por endocrinologistas, clínicos e cirurgiões gerais podem ter sido diferentes. O próprio desenho do estudo impediu algum tipo de análise estatística deste fato. Portanto, foi apenas descrito o número de pacientes que realizaram investigação laboratorial mínima, conforme Kaplan (20): dosagem sérica de potássio, cortisol (após lmg de dexametasona) e S-DHEA, bem como de metanefrinas urinárias.

A medida da densidade da lesão após a administração intravenosa do meio de contraste iodado deve ser utilizada com cautela. Além da espessura do 
corte, o tamanho e a localização do cursor de medida da densidade (region of interest-ROI), a velocidade de infusão do meio de contraste, o tempo de início dos cortes e as próprias condições hemodinâmicas do paciente são fatores que influenciam a estimativa da densidade pós-contraste e a tornam sujeita a muitas variações.

O tipo de impregnação ao meio de contraste e os contornos da lesão adrenal são dados subjetivos e de aplicabilidade clínica discutível. A velocidade de desimpregnação (washout) ao meio de contraste intravenoso tem sido atualmente valorizada para a definição da natureza da lesão, se benigna ou maligna (21-24), porém, pelo próprio desenho do estudo, não foi avaliada nesta amostra.

Russi e cols. (17) e Arnaldi e cols. (1) encontraram maior prevalência de IA nos pacientes com HAS, DM e obesidade. No presente estudo, dispõe-se apenas do número de pacientes com IA que apresentavam estas entidades, pois não foi realizada a revisão dos registros médicos dos demais pacientes.

É bem conhecida a diferença encontrada na interpretação de exames radiológicos de um mesmo médico em momentos distintos. Aspectos subjetivos e a e xperiência adquirida na interpretação das imagens adrenais no transcorrer do estudo talvez expliquem os valores de kappa encontrados, caracterizando uma concordância intra-observador inferior a interobservador.

No que se refere ao diagnóstico final, dos 83 casos de IA, as diversas equipes assistenciais estabeleceram diagnóstico final em $68(82 \%)$, sendo 30 $(36,1 \%)$ com confirmação histológica e em 38 (45,8\%) diagnóstico clínico-laboratorial, predominando nas duas situações as lesões metastáticas e os adenomas. Estes achados são diferentes de estudos que demonstram a predominância dos adenomas $(1,8,14)$, muito embora a importância das lesões metastáticas também seja ressaltada especialmente em pacientes portadores de neoplasia conhecida $(3,25)$. $\mathrm{O}$ achado de que em 15 pacientes ( $18 \%)$ não se obteve diagnóstico final até a conclusão do estudo, reflete, por um lado, a dificuldade diagnóstica que alguns casos apresentam e, por outro, a pouca importância que o achado desencadeia no processo assistencial de equipes não especializadas de um hospital geral. Neste sentido, devemos observar que em muitos destes casos, o achado do IA estava de tal forma dissociado do quadro clínico do paciente que, após investigação sumária, seu diagnóstico final foi protelado para depois da doença de base. Entre os diagnósticos finais, cabe ressaltar ainda os 4 casos de tuberculose e os 3 casos de feocromocitoma. A prevalência de feocromocitoma é semelhante à de algumas séries da literatura $(1,8,14)$, demonstrando a importância deste diagnóstico também em nosso meio, principalmente por exigir investigação cuidadosa e manejo cirúrgico específico, sem os quais os riscos cirúrgicos e anestésicos estão aumentados. No que se refere à tuberculose, o achado decorre, infelizmente, da alta prevalência desta moléstia infecto-contagiosa em nosso meio, chamando a atenção para que a mesma seja incluída no diagnóstico do IA, e também para a possibilidade de desenvolvimento de insuficiência adrenal de causa infecciosa no acompanhamento destes pacientes em nosso meio. Em resumo, observou-se que as lesões adrenais incidentais detectadas prospectivamente por TC são relativamente freqüentes, sendo mais comuns em homens e em faixas etárias mais elevadas. Estes achados ocorrem tanto em TCs de abdome quanto de tórax. Frente ao crescente número de diagnósticos de incidentaloma adrenal e suas possíveis implicações, considerou-se que a notificação da sua prevalência e de suas causas é de significativa importância, especialmente em Endocrinologia, uma vez que sua abordagem deverá motivar um maior intercâmbio com especialistas das áreas de Medicina Interna, Radiologia, Patologia e Cirurgia, entre outras, a fim de ampliar as possibilidades diagnósticas e determinar padronizações de condutas que beneficiem pacientes e reduzam os riscos do manejo deste distúrbio em nosso meio.

\section{REFERÊNCIAS}

1. Arnaldi G, Masini AM, Giacchetti G, Taccaliti A, Faloia E, Mantero F. Adrenal incidentaloma. Braz J Med Biol Res 2000;33:1 177-89.

2. Barzon L, Sonino N, Fallo F, Palu G, Boscaro M. Prevalence and natural history of adrenal incidentalomas. Eur J Endocrinol 2003;149:273-85.

3. Grumbach MM, Biller BM, Braunstein GD, Campbell KK, Carney JA, Godley PA, et al. NIH Conferences: Management of Clinically Inapparent Adrenal Mass ("Incidentaloma"). Ann Intern Med 2003;138:424-9.

4. Abecassis M, McLoughlin MJ, Langer B, Kudlow JE. Serendipitous adrenal masses: prevalence, significance, and management. Am J Roentgenol 1985; 149:783-8.

5. Brunt LM, Moley JF. Adrenal incidentaloma. World $\mathbf{J}$ Surg $2001 ; 25: 905-13$.

6. HCPA. Hospital de Clínicas de Porto Alegre. Área física. Disponivel em: http://www.hcpa.ufrgs.br. Acessado em 23 out, 2004.

7. Kasperlik-Zaluska AA, Roslonowska E, SlowinskaSrzednicka J, et al. Incidentally discovered adrenal mass (incidentaloma): investigation and management 
of 208 patients. Clin Endocrinol 1997;46:29-37.

8. Ferreira JG, Kater CE, Faiçal S, Silva RC, Ajzen S, Borri ML, et al. Clinical, biochemical and pathological findings in a series of adrenal incidentalomas. Ara Bras Endocrinol Metab 1997;41:125-30.

9. Barzon L, Scaroni C, Sonino N, Fallo F, Paoletta A, Boscaro M. Risk factors and long-term follow-up of adrenal incidentalomas. J Clin Endocrinol Metab 1999:84:520-6.

10. Mantero F, Terzolo M, Arnaldi G, Osella G, Masini AM, Ali $A$, et al. A survey of adrenal incidentaloma in Italy. $\mathbf{J}$ Clin Endocrinol Metab 2000;85:637-44.

11. Angeli A, Terzolo M. Adrenal incidentaloma - A modern disease with old complications. J Clin Endocrinol Metab 2002;87:4869-71.

12. Libè R, Dall'Asta C, Barbetta L, Baccarelli A, BeckPeccoz P, Ambrosi B. Long-term follow-up study of patients with adrenal incidentaloma. Eur $\mathbf{J}$ Endocrinol 2002; 147:489-94.

13. Kloos RT, Gross MD, Francis IR, Korobkin M, Shapiro B. Incidentally discovered adrenal masses. Endocr Rev $1995 ; 16: 460-84$.

14. Cook DM, Loriaux DL. The incidental adrenal mass. Am J Med 1996;101:88-94.

15. Chidiac RM, Aron DC. Incidentalomas. A disease of modern technology. Endocrinol Metab Clin North Am 1997:26:233-53

16. Lockhart ME, Smith JK, Kenney PJ. Imaging of adrenal masses. Eur J Radiol 2002;41(2):95-112.

17. Russi S, Blumenthal HT, Gray SH. Small adenomas of the adrenal cortex in hypertension and diabetes. Arch Intern Med 1945;76:284-91.

18. Page DL, DeLellis RA, Hough AJ. Tumors of the adrenal. Washington:AFIP - Armed Forces Institute of Pathology, 1986.p.73-8.

19. Yamada T, Ishibashi T, Saito H, Matsuhashi T, Majima K, Tsuda $M$, et al. Adrenal adenomas: relationship between histologic lipid-rich cells and CT attenuation number. Eur J Radiol 2003;48(2):198-202.

20. Kaplan NM. The adrenal incidentaloma. Available from: http://www.uptodate.com. Acess in May 10, 2000.

21. Heinz-Peer G, Honigschnabl S, Schneider B, Niederle B, Kaserer K, Lechner $G$. Characterization of adrenal masses using MR imaging with histopathologic correlation. Am J Roentgenol 1999; 173:15-22.

22. Peña CS, Boland GW, Hahn PF, Lee MJ, Mueller PR. Characterization of indeterminate (lipid-poor) adrenal masses: use of washout characteristics at contrastenhanced CT. Radiology 2000;217:798-802.

23. Caoili EM, Korobkin M, Francis IR, Cohan RH, Platt JF, Dunnick KR, et al. Adrenal masses: characterization with combined unenhance and delayed enhanced CT. Radiology 2002;222:629-33.

24. Korobkin M. CT characterization of adrenal masses: the time has come. Radiology 2000;217:629-32.

25. Frilling A, Tecklenborg K, Weber F, Kuhl H, Muller S, StamatisG, et al. Importance of adrenal incidentaloma in patients with a history of malignancy. Surgery 2004;136(6): 1289-96.

\section{Endereço para correspondência}

Eleci V. Ferreira

Rua Santo Inácio 510/201

90570-150 Porto Alegre, RS

E-mail: elecivaz@terra.com.br 